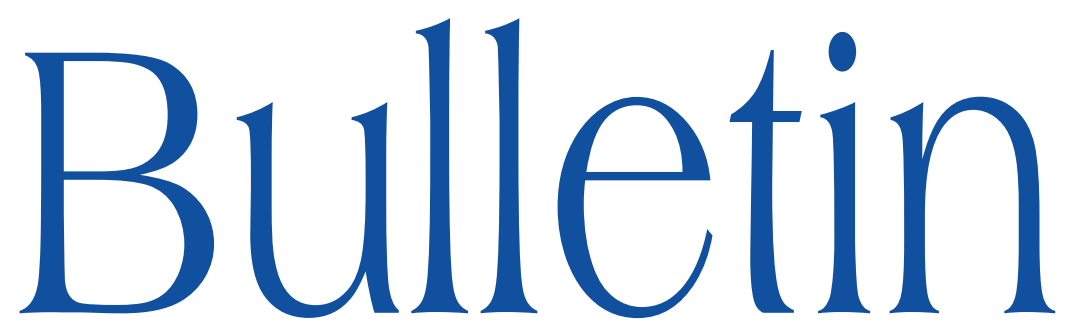

de la SOCIÉTÉ MATHÉMATIQUE DE FRANCE

\title{
ON THE LOOP HOMOLOGY OF COMPLEX PROJECTIVE SPACES
}

David Chataur \& Jean-François Le Borgne

\section{Tome 139}

Fascicule 4

2011 


\title{
ON THE LOOP HOMOLOGY OF COMPLEX PROJECTIVE SPACES
}

\author{
By David Chataur \& Jean-François Le Borgne
}

\begin{abstract}
In this short note we compute the Chas-Sullivan BV-algebra structure on the singular homology of the free loop space of complex projective spaces. We compare this result with computations in Hochschild cohomology.

RÉSUMÉ (Sur l'homologie des lacets d'espaces projectifs complexes)

Sur l'homologie de l'espace des lacets des espaces projectifs complexes résumé : Dans cette note, on calcule l'homologie singulière de l'espace des lacets libres des espaces projectifs complexes munie de la BV-structure de Chas-Sullivan. On compare ces calculs avec ceux effectués en cohomologie de Hochschild.
\end{abstract}

\section{Introduction}

Let us begin by recalling the definitions of Gerstenhaber algebras and $B V$ algebras. A Gerstenhaber algebra is a triple $(A, \bullet,\{-,-\})$ such that $(A, \bullet)$ is a

Texte reçu le 25 mai 2009, révisé le 4 novembre 2010 et le 17 avril 2011, accepté le 23 juin 2011.

David Chataur, Laboratoire Paul Painlevé, Université de Lille 1, 59655 Villeneuve d'Ascq Cedex, France • E-mail : David.Chataur@math.univ-lille1.fr

JeAn-François Le Borgne, Laboratoire Paul Painlevé, Université de Lille 1, 59655 Villeneuve d'Ascq Cedex, France • E-mail : Leborgne. Jean-Francois@math.univ-lille1.fr 2000 Mathematics Subject Classification. — 55P50, 55N45, 55P48.

Key words and phrases. - Free loop spaces, Hochschild homology, string topology.

The second author is partially supported by ANR grant 06-JCJC-0042 "Opérades, Bigèbres et Théories d'Homotopie". 
commutative graded algebra and $(A,\{-,-\})$ is a graded Lie algebra of degree +1 . Moreover the product and the Lie bracket satisfy the following relation the so-called Poisson relation:

$$
\{a, b \bullet c\}=\{a, b\} \bullet c+(-1)^{(|a|+1)|b|} b \bullet\{a, c\} .
$$

A Batalin-Vilkovisky algebra or BV-algebra is a Gerstenhaber algebra $(A, \bullet,\{-,-\})$ equipped with a degree +1 linear map

$$
\Delta: A_{i} \rightarrow A_{i+1}
$$

such that $\Delta^{2}=0$ and such that we have the formula

$$
\{a, b\}=(-1)^{|a|}\left(\Delta(a \bullet b)-\Delta(a) \bullet b-(-1)^{|a|} a \bullet \Delta(b)\right),
$$

these algebras are closely related to topological field theories [9].

The first examples of Gerstenhaber algebras go back to Gerstenhaber himself. Let $A$ be an associative algebra, Gerstenhaber proved that the Hochschild cohomology of $A$ denoted by $H H^{*}(A, A)$ is a Gerstenhaber algebra [8]. L. Menichi [13] and T. Tradler [18] proved that for any symmetric algebra $A$ the Hochschild cohomology $H H^{*}(A, A)$ is a Batalin-Vilkovisky algebra. In this case the $\Delta$-operator is induced by the Connes coboundary map. This result has been reproved and extended by many people. In particular, L. Menichi proved in [14] that the Hochschild cohomology $H^{*}(A, A)$ of a differential graded algebra $A$ which is a symmetric algebra at the level of the derived category of algebras is a Batalin-Vilkovisky algebra. Let $M$ be a $d$-dimensional connected closed oriented manifold and $C^{*}(M, \mathbb{Z})$ its singular cochain complex, in [7] the authors proved a linear isomorphism

$$
D: H H^{*+d}\left(C^{*}(M, \mathbb{Z}), C_{*}(M, \mathbb{Z})\right) \rightarrow H H^{*}\left(C^{*}(M, \mathbb{Z}), C^{*}(M, \mathbb{Z})\right) .
$$

This linear isomorphism depends on choices of $C^{*}(M, \mathbb{Z})$-bimodules quasiisomorphisms between chains and cochains of $M$. And it is required that these quasi-isomorphisms induce the Poincaré duality at the homology level. L. Menichi's results imply that the Connes coboundary map on Hochschild cohomology $H H^{*}\left(C^{*}(M, \mathbb{Z}), C_{*}(M, \mathbb{Z})\right)$ defines via the isomorphism $D$ a structure of BV algebra extending the Gerstenhaber algebra $H H^{*}\left(C^{*}(M, \mathbb{Z}), C^{*}(M, \mathbb{Z})\right)$.

The second examples of BV-algebras we encounter in this note come from string topology. The free loop space of $M$ denoted by $\mathscr{L} M$ is the space of continuous maps from the circle $S^{1}$ to $M$. In [3], M. Chas and D. Sullivan defined on the shifted homology $\mathbb{H}_{*}(\mathscr{L} M):=H_{*+d}(\mathscr{L} M, \mathbb{Z})$ a structure of BV algebra. The commutative product is the loop product denoted by $\circ$. This loop product is a kind of intersection product for free loop spaces of manifolds. It mixes the intersection product of the singular homology of $M$ together with the Pontryagin product of the homology of the based loop space $\Omega_{m} M$. The $\Delta$-operator comes from the natural action of the circle on $\mathcal{L} M$ given by reparametrization

TOME $139-2011-\mathrm{N}^{\mathrm{O}} 4$ 
of loops. More precisely, this operator is the composition of the two following maps. First, we consider the morphism

$$
\begin{gathered}
{\left[S^{1}\right] \times: H_{*}(\mathscr{L} M) \rightarrow H_{*+1}\left(S^{1} \times \mathscr{L} M\right)} \\
x \mapsto\left[S^{1}\right] \times x
\end{gathered}
$$

given by the cross product with the fundamental class of $S^{1}$. Next, we consider

$$
\operatorname{act}_{*}: H_{*+1}\left(S^{1} \times \mathscr{L} M\right) \rightarrow H_{*+1}(\mathscr{L} M)
$$

the map induced in homology by the reparametrization action of the circle:

$$
\begin{gathered}
\text { act }: S^{1} \times \mathscr{L} M \rightarrow \mathscr{L} M \\
(\theta, \gamma(t)) \mapsto \gamma(\theta+t) .
\end{gathered}
$$

For more details on the constructions of the loop product and of the string topology BV-structure we refer to Chas-Sullivan and Cohen-Jones'papers ([3] and [4]).

A fundamental problem in string topology is to compare $(\mathbb{H}(\mathscr{L} M), \circ, \Delta)$ together with $H H^{*}\left(C^{*}(M, \mathbb{Z}), C^{*}(M, \mathbb{Z})\right)$. Working over a field $\mathbb{F}$, in [4] Cohen and Jones proved an isomorphism of graded commutative and associative algebras

$$
\mathbb{H}_{*}(\mathscr{L} M, \mathbb{F}) \cong H H^{*}\left(C^{*}(M, \mathbb{F}), C^{*}(M, \mathbb{F})\right) .
$$

Now, let us suppose that $M$ is a formal manifold, that is to say that $C^{*}(M, \mathbb{Z})$ and $H^{*}(M, \mathbb{Z})$ are isomorphic in the homotopy category of differential graded algebras. For example, spheres and projective spaces are formal manifolds. In that cases, in first approximation one can use the structure of Poincaré duality algebra of $H^{*}(M, \mathbb{Z})$ and try to compare $\left(\mathbb{H}_{*}(\mathscr{L} M), \circ, \Delta\right)$ together with $H H^{*}\left(H^{*}(M, \mathbb{Z}), H^{*}(M, \mathbb{Z})\right)$ as BV-algebras. For the manifold $M=\mathbb{C} P^{1}=S^{2}$, L. Menichi in [15] proved that $\left(\mathbb{H}_{*}\left(\mathcal{L} S^{2}, \mathbb{Z} / 2 \mathbb{Z}\right), \circ, \Delta\right)$ cannot be isomorphic to $H H^{*}\left(H^{*}\left(S^{2}, \mathbb{Z} / 2 \mathbb{Z}\right), H^{*}\left(S^{2}, \mathbb{Z} / 2 \mathbb{Z}\right)\right)$ as a BV-algebra. Then L. Menichi asked for the case of complex projective spaces. It is the aim of this paper to give these computations. We prove the existence of an isomorphism of BV-algebras for $M=\mathbb{C} P^{2 m}$ but never for $M=\mathbb{C} P^{2 m+1}$, however we always have an isomorphism of Gerstenhaber algebras. All our computations are with homology with integral coefficients, we notice that few computations of loop homology BV-algebras were done over the integers, H. Tamanoi in [17] did it for complex Stiefel manifolds. These computations prove that in string topology the comparison of the Chas-Sullivan BV-algebra with Hochschild cohomology of cochains is very subtle. In fact the main point is to produce a correct notion of homotopy Poincaré duality algebra. And maybe for such a notion complex projective spaces of odd dimension are not formal manifolds. Recently $R$. Hepworth in [11] has also computed the Chas-Sullivan BV-algebra of complex projective spaces by using different methods. 


\section{Statements and results}

Chas-Sullivan BV structure. - In [6], the authors proved that rationaly for 1-connected closed oriented manifolds, there is an isomorphism of BV algebra betwen the Hochschild cohomology $H H_{*}\left(C^{*}(M, \mathbb{Q}), C^{*}(M, \mathbb{Q})\right)$ and $\left(\mathbb{H}_{*}(\mathscr{L} M, \mathbb{Q}), \circ, \Delta\right)$. As a consequence of this isomorphism T. Yang deduced that $([19$, theorem 4.3$])$

As $B V$ algebras,

$$
\mathbb{H}_{*}\left(\mathscr{L} \mathbb{C} P^{n} ; \mathbb{Q}\right)=\mathbb{Q}\left[\alpha_{-2}, \xi_{-1}, \zeta_{2 n}\right] /\left(\alpha_{-2}^{n+1}, \xi_{-1}^{2}, \alpha_{-2}^{n} \xi_{-1}, \zeta_{2 n} \alpha_{-2}^{n}\right)
$$

where the subscripts are the degrees of the generator.

$$
\begin{gathered}
\Delta\left(\zeta_{2 n}^{k} \alpha_{-2}^{l}\right)=0 \\
\Delta\left(\zeta_{2 n}^{k} \xi_{-1} \alpha_{-2}^{l}\right)=(-(k+1) n-k+l) \zeta_{2 n}^{k} \alpha_{-2}^{l}
\end{gathered}
$$

In [5], the authors compute the loop algebra $\mathbb{H}_{*}\left(\mathscr{L} \mathbb{C} P^{n}, \mathbb{Z}\right)$. Their computation uses the Leray-Serre spectral sequence of the evaluation fibration

$$
\Omega_{m} \mathbb{C} P^{n} \rightarrow \mathscr{L} \mathbb{C} P^{n} \stackrel{e v_{0}}{\rightarrow} \mathbb{C} P^{n} .
$$

They used the fact that this fibration is multiplicative with respect to the loop product and showed that

The algebra $\mathbb{H}_{*}\left(\mathscr{L} \mathbb{C} P^{n}\right)$ is isomorphic to

$$
\mathbb{Z}\left[a_{-2}, x_{-1}, y_{2 n}\right] /\left(a_{-2}^{n+1}, x_{-1}^{2}, a_{-2}^{n} x_{-1},(n+1) y_{2 n} a_{-2}^{n}\right)
$$

where the subscripts are the degrees of the generators.

One can already notice that Cohen-Jones-Yan's computations rely upon W. Ziller's results [20] that are based on Morse theoretic arguments.

Using Morse theory we prove that:

THEOREM 0.1. - The BV structure on

$$
\mathbb{H}_{*}\left(\mathcal{L} \mathbb{C} P^{n}\right) \simeq \mathbb{Z}\left[a_{-2}, x_{-1}, y_{2 n}\right] /\left(a_{-2}^{n+1}, x_{-1}^{2}, a_{-2}^{n} x_{-1},(n+1) y_{2 n} a_{-2}^{n}\right)
$$

is given by

$$
\Delta\left(y_{2 n}^{k} a_{-2}^{l}\right)=0
$$

For $l \neq 0$,

$$
\Delta\left(y_{2 n}^{k} x_{-1} a_{-2}^{l}\right)=(-(k+1) n-k+l) y_{2 n}^{k} a_{-2}^{l}
$$

If $n$ is odd,

$$
\Delta\left(y_{2 n}^{k} x_{-1}\right)=(-(k+1) n-k) y_{2 n}^{k}+(k+1) \cdot \frac{n+1}{2} a_{-2}^{n} y_{2 n}^{k+1} .
$$

If $n$ is even,

$$
\Delta\left(y_{2 n}^{k} x_{-1}\right)=(-(k+1) n-k+l) y_{2 n}^{k} .
$$

TOME $139-2011-\mathrm{N}^{\mathrm{O}} 4$ 
The first step of this computation involves the Morse spectral sequence associated to the energy filtration. The filtration of loops by the energy leads at least for rank 1 symmetric spaces to a spectral sequence that is multiplicative with respect to the loop product. The $E^{1}$ page of this spectral sequence depends of the intersection homology of $\mathbb{C} P^{n}$ and of the intersection homology of $U \mathbb{C} P^{n}$ which is the unit tangent bundle of $\mathbb{C} P^{n}$. The second step of the proof is the computation of the operator $\Delta$ on each column of this spectral sequence. We compute the action of $\Delta$ on $H_{*}\left(U \mathbb{C} P^{n}\right)$. In order to finish this computation we consider the action of $\Delta$ on the torsion free part of $\mathbb{H}_{*}\left(\mathscr{L} \mathbb{C} P^{n}\right)$ given by the rational homotopy theoretic computations of Félix-Thomas and Yang.

Comparison with Hochschild cohomology. - The cohomology of $\mathbb{C} P^{n}$ is isomorphic to the graded algebra $\mathbb{Z}\left[a_{-2}\right] /\left(a_{-2}^{n+1}\right)$. It is the cohomology ring of a closed oriented manifold, thus it is a symmetric algebra. Based on the study of Hochschild cohomology of symmetric algebras cited previously, in [19] T. Yang has determined the BV-algebra structure on

$$
H H^{*}\left(\mathbb{Z}\left[a_{-2}\right] /\left(a_{-2}^{n+1}\right), \mathbb{Z}\left[a_{-2}\right] /\left(a_{-2}^{n+1}\right)\right)
$$

an thus on $H H^{*}\left(H^{*}\left(\mathbb{C} P^{n}, \mathbb{Z}\right), H^{*}\left(\mathbb{C} P^{n}, \mathbb{Z}\right)\right)$ this structure is given by the Poincaré duality isomorphism $H^{*}\left(\mathbb{C} P^{n}, \mathbb{Z}\right) \cong H_{2 n-*}\left(\mathbb{C} P^{n}, \mathbb{Z}\right)$. Let us call this structure the formal BV-structure. In that case we have the following result

The formal $B V$ structure on

$H H^{*}\left(H^{*}\left(\mathbb{C} P^{n}, \mathbb{Z}\right), H^{*}\left(\mathbb{C} P^{n}, \mathbb{Z}\right)\right) \cong \mathbb{Z}\left[a_{-2}, x_{-1}, y_{2 n}\right] /\left(a_{-2}^{n+1}, x_{-1}^{2}, a_{-2}^{n} x_{-1},(n+1) y_{2 n} a_{-2}^{n}\right)$

is given by

$$
\Delta_{f}\left(y_{2 n}^{k} a_{-2}^{l}\right)=0
$$

and

$$
\Delta_{f}\left(y_{2 n}^{k} x_{-1} a_{-2}^{l}\right)=(-(k+1) n-k+l) y_{2 n}^{k} a_{-2}^{l} .
$$

Thus we get the following comparison theorem:

TheOREM 0.2. - 1) For $\mathbb{C} P^{2 m}$ the Chas-Sullivan BV-structure is isomorphic to the formal $B V$-structure, whereas it is never the case for $\mathbb{C} P^{2 m+1}$. But we always have a Gerstenhaber algebra isomorphism.

2) For $\mathbb{C} P^{2 n+1}$ we have a $B V$-isomorphism when working with coefficients in the ring $\mathbb{Z}\left[\frac{1}{2 n+2}\right]$.

3) Over $\frac{\mathbb{Z}}{2 \mathbb{Z}}$ the Chas-Sullivan $B V$-structure of the homology of $\mathscr{L} \mathbb{C} P^{4 n+1}$ can not be isomorphic to the formal BV-structure. 
Proof. - 1) If $n$ is even, the Chas-Sullivan BV-algebra $\mathbb{H}_{*}\left(\mathscr{L} \mathbb{C} P^{n}, \mathbb{Z}\right)$ and the formal BV-algebra $H H^{*}\left(H^{*}\left(\mathbb{C} P^{n}, \mathbb{Z}\right), H^{*}\left(\mathbb{C} P^{n}, \mathbb{Z}\right)\right)$ are the same and 1$)$ is obvious. Suppose that $n$ is odd. Let

$$
\phi: H H^{*}\left(H^{*}\left(\mathbb{C} P^{n}, \mathbb{Z}\right), H^{*}\left(\mathbb{C} P^{n}, \mathbb{Z}\right)\right) \rightarrow \mathbb{H}_{*}\left(\mathscr{L} C P^{n}, \mathbb{Z}\right)
$$

be an isomorphism of algebras. Since $\phi$ is an isomorphism in degree -1 , $\phi\left(x_{-1}\right)= \pm x_{-1}$. And since $\phi$ is an isomorphism of algebras $\phi \Delta_{f}\left(x_{-1}\right)=$ $\phi(-n .1)=-n .1$. Since $\mathbb{H}_{0}\left(\mathcal{L} \mathbb{C} P^{n}, \mathbb{Z}\right) \cong \mathbb{Z}<1>\oplus \frac{\mathbb{Z}}{n+1 \mathbb{Z}}<a_{-2}^{n} y_{2 n}>$ and since

$$
\Delta\left(\phi\left(x_{-1}\right)\right)= \pm\left(-n 1+\frac{n+1}{2} a_{-2}^{n} y_{2 n}\right)
$$

we have $\phi\left(\Delta_{f}\left(x_{-1}\right)\right) \neq \Delta\left(\phi\left(x_{-1}\right)\right)$. An easy computation shows the two corresponding Gerstenhaber algebras are the same.

2) It is a direct consequence of the Kunneth isomorphism and the fact that for any commutative ring $R$ the canonical map

$$
i: \mathbb{H}_{*}(\mathscr{L} M, \mathbb{Z}) \rightarrow \mathbb{H}_{*}(\mathscr{L} M, R)
$$

is a morphism of BV-algebra (see [15]).

3) We compute $\mathbb{H}_{i}\left(\mathscr{L} \mathbb{C} P^{4 n+1}, \frac{\mathbb{Z}}{2 \mathbb{Z}}\right)$ for $i=-1$ and $i=0$, a direct application of the universal coefficient theorem shows that for $i=-1,0$ we have

$$
\mathbb{H}_{i}\left(\mathscr{L} \mathbb{C} P^{4 n+1}, \frac{\mathbb{Z}}{2 \mathbb{Z}}\right) \cong \mathbb{H}_{i}\left(\mathscr{L} \mathbb{C} P^{4 n+1}, \mathbb{Z}\right) \otimes \frac{\mathbb{Z}}{2 \mathbb{Z}}
$$

Thus we have

$$
\mathbb{H}_{-1}\left(\mathscr{L} \mathbb{C} P^{4 n+1}, \frac{\mathbb{Z}}{2 \mathbb{Z}}\right) \cong \frac{\mathbb{Z}}{2 \mathbb{Z}}<x_{-1}>
$$

and in degree zero

$$
\mathbb{H}_{0}\left(\mathscr{L} \mathbb{C} P^{4 n+1}, \frac{\mathbb{Z}}{2 \mathbb{Z}}\right) \cong \frac{\mathbb{Z}}{2 \mathbb{Z}}<1>\oplus \frac{\mathbb{Z}}{2 \mathbb{Z}}<a_{-2}^{n} y_{2 n}>
$$

the arguments used to prove assertion 1) remain valid because the operators commute with reduction mod 2 , and because modulo 2 we have $\Delta_{f}\left(x_{-1}\right)=1$ and $\Delta\left(x_{-1}\right)=1+a_{-2}^{n} y_{2 n}$.

\section{The Morse spectral sequence for $\mathbb{C} P^{n}$}

Let us recall the basics of Morse theory for free loop spaces, we refer the reader to Bott's survey paper [1]. N. Hingston and M. Goresky have studied the compatibility of the loop product together with the energy fitration in [10]. In this section we follow F. Laudenbach's presentation [12]. Let $M$ be a 1connected rank 1 symmetric space. In that case all the geodesics of $M$ are closed, moreover we suppose that their length is equal to 1 . We denote by $\Lambda$ the space of loops parametrized proportionnaly to arc-length, and we work 
with a completion of the space of piecewise-smooth loops. We recall that the inclusion

$$
\Lambda \rightarrow \mathscr{L} M
$$

is a homotopy equivalence. Let $l^{2}: \Lambda \rightarrow \mathbb{R}$ be the square-length. This function satisfies the Morse-Bott non-degeneracy condition. Let $p \in \mathbb{N}$, we consider the space $\Lambda_{p} \subset \Lambda$ of loops of length $\leq p$ and $\Sigma_{p}$ the space of geodesics of length $p$. We have a geometric filtration

$$
M=\Lambda_{0} \subset \Lambda_{1} \subset \cdots \subset \Lambda_{p} \subset \cdots \subset \Lambda .
$$

We define a filtration

$$
F_{p} C_{p+q}(\Lambda):=C_{p+q}\left(\Lambda_{p}\right) .
$$

This filtration yields a spectral sequence called the Morse spectral sequence. We remark that as the $S^{1}$-action preserves the length of the loops we have the following result :

Proposition 1.1. - The operator $\Delta$ is compatible with the length filtration namely $\Delta\left(C_{p+q}\left(\Lambda_{p}\right)\right) \subset C_{p+q+1}\left(\Lambda_{p}\right)$.

The first page. - We identify the $E^{1}$-term of the spectral sequence together with the action of the operator $\Delta$. We first remark that the elements of $\Sigma_{p}$ are the primitive geodesics traversed $p$ times. They are the critical points of $l^{2}$ of index $\alpha_{p}$ (for $\left.p>0\right)$. In order to compute the $E^{1}$-term one needs to determine the relative groups

$$
H_{*}\left(\Lambda_{p}, \Lambda_{p-1}, \mathbb{Z}\right),
$$

it follows from Morse theory that there is a $S^{1}$-equivariant attaching map $f$ : $\Sigma_{p} \rightarrow \Lambda_{p-1}$ and that we have

$$
\Lambda_{p} \cong \Lambda_{p-1} \cup_{f} \Sigma_{p}
$$

The pair $\left(\Lambda_{p}, \Lambda_{p-1}\right)$ is $S^{1}$-equivariantly homotopy equivalent to a pair $\left(D_{p}, S_{p}\right)$. The space $D_{P}$ (resp. $\left.S_{P}\right)$ is the disk bundle (resp. the sphere bundle) of a $S^{1}$ vector bundle

$$
\mu_{p}^{-} \rightarrow \Sigma_{p}
$$

over $\Sigma_{p}([16]$, sections 7 and 8$)$. Associated to this bundle we have Thom's isomorphism $\phi_{p}$ for $p>0$

$$
\phi_{p}: H_{*}\left(\Sigma_{p}, \mathbb{Z}\right) \rightarrow H_{*+\alpha_{p}}\left(D_{p}, S_{p}\right) \cong H_{*+\alpha_{p}}\left(\Lambda_{p}, \Lambda_{p-1}\right) .
$$

We recall that the index $\alpha_{p}$ satisfies Bott's iteration formula

$$
\alpha_{p}=p \alpha_{1}+(p-1)(d-1) .
$$


We have a $\Delta$ operator on $H_{*}\left(\Sigma_{p}, \mathbb{Z}\right)$ coming from the natural $S^{1}$-action on iterated geodesics, the relative homology groups $H_{*+\alpha_{p}}\left(D_{p}, S_{p}\right)$ are also equipped with a $\Delta$-operator because

$$
\mu_{p}^{-} \rightarrow \Sigma_{p}
$$

is a $S^{1}$-vector bundle and the action is compatible with the metric. From the existence of $S^{1}$-Thom spaces (see [16], section 5) and equivariant Thom classes it follows that $\phi_{p}$ respects the $\Delta$-operators. Let $U M$ be the unit tangent bundle of $M$, we have a diffeomorphism

$$
\begin{gathered}
D: \Sigma_{p} \rightarrow U M \\
\gamma \mapsto\left(\gamma(0), \gamma^{\prime}(0) /\left\|\gamma^{\prime}(0)\right\|\right) .
\end{gathered}
$$

which equips $U M$ with $p$-iterated $S^{1}$-actions. We conclude that the computation of $\Delta$ on the first term of the spectral sequence follows from the determination of $\Delta$ on the homology of $\Sigma_{p}$.

Algebra structure. - Now, we describe its multiplicative structure. Let us denote by $\mathbb{H}_{*}(M):=\mathbb{H}_{*+d}(M, \mathbb{Z})$ and $\mathbb{H}_{*}(U M):=H_{*+2 d-1}(U M, \mathbb{Z})$ the intersection algebras of $M$ and $U M$. We introduce a bigraded algebra $\mathscr{C}_{*, *}$. We set

$$
\mathscr{G}_{0, q}:=\mathbb{H}_{q}(M)
$$

and

$$
\mathscr{G}_{p, q}:=\mathbb{H}_{p+q-\alpha_{p}}(U M)<T^{p}>
$$

if $p>0$ where $T$ is an element of bidegree $\left(1, \alpha_{1}+d-2\right)$. The multiplicative structure of $\mathscr{G}_{*, *}$ is given by :

1) the intersection algebra $\mathbb{H}_{*}(M)$ if $p=0$,

2) the algebra $\mathbb{H}_{p+q-\alpha_{p}}(U M)[T]_{\geq 1}$ of polynomials of degree $\geq 1$ in $T$ with ocefficients in the intersection algebra $\mathbb{H}_{*}(U M)$ when $p>0$,

$3)$ the products involving an element of $\mathbb{H}_{*}(M)$ and an element of $\mathbb{H}_{p+q-\alpha_{p}}(U M)[T]_{\geq 1}$ is given by a topological $\mathbb{H}_{*}(M)$-module structure on $\mathbb{H}_{*}(U M)$. This structure is given by the Gysin map

$$
p_{!}: \mathbb{H}_{*}(M) \rightarrow \mathbb{H}_{*}(U M)
$$

associated to the canonical projection $p: U M \rightarrow M$, the morphism $p_{\text {! }}$ is a morphism of intersection algebra. Then at the homological level the module structure is given by the formula

$$
\begin{gathered}
\mathbb{H}_{u}(M) \otimes \mathbb{H}_{v}(U M) T^{k} \rightarrow \mathbb{H}_{u+v}(U M) T^{k} \\
x \otimes y T^{k} \mapsto\left(p_{!}(x) \bullet y\right) T^{k} .
\end{gathered}
$$

We are now ready to recall the main computational tool (see [10] and [12]) used in this note. 
THEOREM 1.2. - If $M$ is a d-dimensional 1-connected rank 1 symmetric space, the Morse spectral sequence is a spectral sequence of algebra converging to the algebra $\left(\mathbb{H}_{*}(\mathscr{L} M), \circ, \Delta\right)$. As algebra, $\mathbb{E}_{*, *}^{1}(\mathcal{M})(\mathscr{L} M)$ is isomorphic to $\mathscr{G}_{*, *}$. In other words, the multiplicative structure on the shifted Morse spectral sequence

$$
\mathbb{E}_{p, q}^{r}(\mathcal{M})(\mathscr{L} M):=E_{p, q+d}^{r}(\mathcal{M})(\mathscr{L} M)
$$

is given at the $E^{1}$-level by:

$$
\mathbb{E}_{*, *}^{1}(\mathcal{M})(\mathscr{L} M)=\mathbb{H}_{*}(M) \oplus \mathbb{H}_{*}(U M)[T]_{\geq 1} .
$$

The bidegree of $T$ is $\left(1, \alpha_{1}+d-2\right)$, elements of $\mathbb{H}_{*}(M)$ and of $\mathbb{H}_{*}(U M)$ are of bidegree $(0, *)$. We have

$$
\mathbb{E}_{0, q}^{1}(\mathcal{M})(\mathscr{L} M)=\mathbb{H}_{q}(M)
$$

and for $p \geq 1$,

$$
\mathbb{E}_{p, q}^{1}(\mathcal{M})(\mathcal{L} M)=\mathbb{H}_{q-p \alpha_{1}}(U M)<T^{p}>
$$

The multiplication between the 0-th column and the others is induced by the $\mathbb{H}_{*}(M)$-module structure on $\mathbb{H}_{*}(U M)$. Moreover this spectral sequence collapses at the first term.

The case of complex projective spaces. - In the case of complex projective spaces we have $\alpha_{1}=1$. Moreover the intersection algebras of $\mathbb{C} P^{n}$ and $U \mathbb{C} P^{n}$ are given by

$$
\mathbb{H}_{*}\left(\mathbb{C} P^{n}, \mathbb{Z}\right) \cong \mathbb{Z}[a] /\left(a^{n+1}\right)
$$

where $\operatorname{deg}(a)=-2$ and

$$
\mathbb{H}_{*}\left(U \mathbb{C} P^{n}, \mathbb{Z}\right) \cong \mathbb{Z}[\alpha, \beta] /\left((n+1) \alpha^{n}, \alpha^{n+1}, \beta^{2}, \alpha^{n} \beta\right)
$$

where $\operatorname{deg}(\alpha)=-2, \operatorname{deg}(\beta)=-2 n-1$. The computation of $\mathbb{H}_{*}\left(U \mathbb{C} P^{n}, \mathbb{Z}\right)$ follows from the Leray-Serre spectral sequence of the fiber bundle

$$
S^{2 n-1} \rightarrow U \mathbb{C} P^{n} \rightarrow \mathbb{C} P^{n}
$$

Description of the first term. - We consider the $E^{1}$-term of the Morse spectral sequence. From the preceding theorem one has that

$$
\mathbb{E}_{*, *}^{1}(\mathcal{M})=\mathbb{Z}[a] /\left(a^{n+1}\right) \oplus \mathbb{Z}[\alpha, \beta] /\left((n+1) \alpha^{n}, \alpha^{n+1}, \beta^{2}, \alpha^{n} \beta\right)[T]_{\geq 1},
$$

where $\operatorname{bideg}(a)=(0,-2), \operatorname{bideg}(\alpha)=(0,-2), \operatorname{bideg}(\beta)=(0,-2 n-1)$ and $\operatorname{bideg}(T)=(1,2 n-1)$. In order to get a complete description of the multiplicative structure we have to give the $\mathbb{H}_{*}\left(\mathbb{C} P^{n}\right)$-module structure on $\mathbb{H}_{*}\left(U \mathbb{C} P^{n}\right)$. Let

$$
p: U \mathbb{C} P^{n} \rightarrow \mathbb{C} P^{n}
$$


be the canonical projection, by a direct application of Poincaré duality and of the Leray-Serre cohomological spectral sequence of $p$ one deduces that $p_{!}(a)=$ $\alpha$. Thus one deduces the isomorphism of algebras

$$
\mathbb{E}_{*, *}^{1}(\mathcal{M}) \cong \mathbb{Z}\left[a_{-2}, x_{-1}, y_{2 n}\right] /\left(a_{-2}^{n+1}, x_{-1}^{2}, a_{-2}^{n} x_{-1},(n+1) y_{2 n} a_{-2}^{n}\right)
$$

where we have identified $a$ with $a_{-2}, \beta T$ with $x_{-1}$ and $T$ with $y_{2 n}$.

The $E^{\infty}$-term. - From W. Ziller's results on the loop homology of free loop spaces of rank 1 symmetric spaces [20], we know that this spectral sequence collapses at the first term, but let us redo the computation for complex projective spaces. To compute the $E^{2}$-term of this spectral sequence we only need to compute the differential $d^{1}$ of the three generators because $d^{1}$ is a derivation (we have a spectral sequence of algebra). If $d^{1}\left(a_{-2}\right)=d^{1}\left(y_{2 n}\right)=0$ for obvious degree reasons we have $d^{1}\left(x_{-1}\right)=l . \alpha_{-2}$, but $l=0$ because $\mathbb{H}_{*}(M)$ is a direct factor of $\mathbb{H}_{*}(\mathscr{L} M)$ (due to the inclusion of constant loops). We deduce that $E^{1}=E^{2}$ and that the derivations $d^{r}=0$ when $r>1$ for degree reasons. Then we have the isomorphism of graded algebras $\mathbb{E}_{*, *}^{1}(\mathcal{M}) \cong \mathbb{E}_{*, *}^{\infty}(\mathcal{M})$.

Extensions issues. - The only possible extension issues are in total degree $2 \mathrm{kn}$, in degree zero we have

$$
\mathbb{E}_{0}^{\infty}=\mathbb{Z} 1 \oplus \mathbb{Z} /(n+1) \mathbb{Z} a_{-2}^{n} y_{2 n}
$$

As $\mathbb{H}_{*}($ evaluation $)\left(a_{-2}^{n} y_{2 n}\right)=0$ and because $\mathbb{H}_{0}(M) \cong \mathbb{Z} .1$ is a direct factor of $\mathbb{H}_{0}(\mathscr{L} M)$ we deduce that $\mathbb{H}_{0}=\mathbb{Z} \oplus \mathbb{Z} /(n+1) \mathbb{Z}$. In degrees $2 k n$ we use the multiplicative structure to conclude. As we have $(n+1)$-torsion in degree zero we get the equation

$$
(n+1) a_{-2}^{n} y_{2 n}^{k+1}=0
$$

thus we have $(n+1)$-torsion in $\mathbb{H}_{2 k n}(\mathscr{L} M, \mathbb{Z})$. The fact that we have no multiplicative extension issues follows for degree reasons, for example $x_{-1}^{2}=0$ because the Chas-Sullivan algebra is a graded commutative algebra.

Thus we have an isomorphism of graded algebra

$$
\mathbb{H}_{*}(\mathscr{L} M, \mathbb{Z}) \cong \mathbb{Z}\left[a_{-2}, x_{-1}, y_{2 n}\right] /\left(a_{-2}^{n+1}, x_{-1}^{2}, a_{-2}^{n} x_{-1},(n+1) y_{2 n} a_{-2}^{n}\right)
$$

We have recovered Cohen-Jones-Yan computation [5].

TOME $139-2011-\mathrm{N}^{\mathrm{O}} 4$ 


\section{The BV-operator}

2.1. On some algebraic considerations. - In this section we follow CadekMoravek's paper [2] where they compute the BV structure for quaternionic projective spaces. We know that $B V$-algebras satisfy the 7 -terms relation

$$
\begin{aligned}
\Delta(a b c)= & \Delta(a b) c+(-1)^{|a|} a \Delta(b c)+(-1)^{(|a|+1)|b|} b \Delta(a c) \\
& -\Delta(a) b c-(-1)^{|a|} a \Delta(b) c-(-1)^{|a|+|b|} a b \Delta(c) .
\end{aligned}
$$

This relation tells us that in order to determine the operator $\Delta$ of a BV algebra we only have to compute it on generators and double product of generators. Moreover for the BV Chas-Sullivan algebra $\Delta$ vanishes on the image of $\mathbb{H}_{*}(M)$ in $\mathbb{H}_{*}(\mathscr{L} M)$, because the $S^{1}$-action is trivial on constant loops. For complex projective spaces we deduce that

$$
\Delta\left(a_{-2}^{k}\right)=0
$$

for any $k$. As we have noticed precedently the $S^{1}$-action respects the filtration, moreover we know that as graded modules

$$
\mathbb{H}_{i}\left(\mathcal{L} \mathbb{C} P^{n}\right) \cong \bigoplus_{p+q=i} \mathbb{E}_{p, q}^{\infty}
$$

thus we get that for any $x \in \mathbb{E}_{p, q}^{\infty}$ we have

$$
\Delta(x) \in \bigoplus_{l+m=p+q+1, l \leq p} \mathbb{E}_{l, m}^{\infty} .
$$

For example if $x \in \mathbb{E}_{1, q}^{\infty}$ then $\Delta(x) \in \mathbb{E}_{1, q+1}^{\infty} \oplus \mathbb{E}_{0, q+2}^{\infty}$. From these considerations by looking at the three first columns of the Morse spectral sequence it follows that

$$
\Delta\left(y_{2 n}^{k} a_{-2}^{l}\right)=0
$$

for any $k$ and $l$. We are left with the following computations :

$$
\Delta\left(x_{-1}\right), \Delta\left(x_{-1} a_{-2}\right), \Delta\left(y_{2 n} x_{-1}\right) .
$$

LEMma 2.1. - We have

$$
\Delta\left(x_{-1}\right)=-n .1+\lambda a_{-2}^{n} y_{2 n}
$$

where $\lambda \in \mathbb{Z} /(n+1) \mathbb{Z}$, and for any $l \neq 0$ we have

$$
\Delta\left(x_{-1} a_{-2}^{l}\right)=-(n-l) a_{-2}^{l} .
$$

Proof. - Let us suppose that $n \geq 2$, we also suppose that

$$
\Delta\left(x_{-1}\right)=\mu_{0} \cdot 1+\lambda a_{-2}^{n} y_{2 n}
$$

and that for $l>0$

$$
\Delta\left(x_{-1} a_{-2}^{l}\right)=\mu_{l} a_{-2}^{l} .
$$


Thanks to the 7-terms relation applied to

$$
\Delta\left(x_{-1} a_{-2}^{l}\right)=\Delta\left(x_{-1} a_{-2} a_{-2}^{l-1}\right)
$$

we get $\mu_{l}=\mu_{1}-\mu_{0}+\mu_{l-1}$. Since $x_{-1} a_{-2}^{n}=0$ we get the equality $\mu_{n}=0$. Moreover we have $n \mu_{1}=(n-1) \mu_{0}$ and $\mu_{0}=n \mu_{n-1}$. In order to determine $\mu_{n-1}$ we consider the canonical inclusion $S^{2} \rightarrow \mathbb{C} P^{n}$ and the induced map $i: \mathscr{L} S^{2} \rightarrow \mathscr{L} \mathbb{C} P^{n}$, in homology we get a morphism

$$
i_{*}: \mathbb{H}_{i}\left(\mathscr{L} S^{2}\right) \cong H_{i+2}\left(\mathscr{L} S^{2}\right) \rightarrow H_{i+2}\left(\mathcal{L} \mathbb{C} P^{n}\right) \cong \mathbb{H}_{i-2(n-1)}\left(\mathcal{L} \mathbb{C} P^{n}\right) .
$$

In degree -1 we have an isomorphism (this can be proved easily by using the Cohen-Jones-Yan spectral sequences associated to $\mathscr{L} S^{2}$ and $\mathscr{L} \mathbb{C} P^{n}$ )

$$
\mathbb{H}_{-1}\left(\mathscr{L} S^{2}\right) \cong \mathbb{Z}<x_{-1}^{\prime}>\cong \mathbb{H}_{-2 n+1}\left(\mathscr{L} \mathbb{C} P^{n}\right) \cong \mathbb{Z}<a_{-2}^{n-1} x_{-1}>
$$

we have $i_{*}\left(x_{-1}^{\prime}\right)=a_{-2}^{n-1} x_{-1}$. We also recall that

$$
\mathbb{H}_{0}\left(\mathcal{L} S^{2}\right) \cong \mathbb{Z}<x_{-1}^{\prime}>\oplus \frac{\mathbb{Z}}{2 \mathbb{Z}}<a_{-2}^{\prime} y_{2}^{\prime}>
$$

we have $i_{*}(1)=a_{-2}^{n-1}$ and $i_{*}\left(a_{-2}^{\prime} y_{2}^{\prime}\right)=0$. Now we use the fact that $i_{*}$ commutes with $\Delta$, as we have $i_{*}\left(\Delta\left(x_{-1}\right)\right)=i_{*}\left(-1+a_{-2}^{\prime} y_{2}^{\prime}\right)=-i_{*}(1)=-a_{-2}^{n-1}$ (see [15] for the computation of $\Delta\left(x_{-1}^{\prime}\right)$ in the case of a 2 -sphere $)$, we get that $\mu_{n-1}=-1$ thus $\mu_{0}=-n$ and $\mu_{1}=-(n-1)$.

LEMMA 2.2. - We have

$$
\Delta\left(y_{2 n} x_{-1}\right)=(-2 n-1) y_{2 n}+\mu a_{-2}^{n} y_{2 n}^{2} .
$$

Proof. - We suppose that $\Delta\left(y_{2 n} x_{-1}\right)=\mu^{\prime} y_{2 n}+\mu a_{-2}^{n} y_{2 n}^{2}$. Let

$$
i: \mathbb{H}_{*}(\mathscr{L} M, \mathbb{Z}) \rightarrow \mathbb{H}_{*}(\mathscr{L} M, \mathbb{Q})
$$

be the canonical map. this map is a morphism of BV-algebras. The rational BV-structure described in the introduction is only determined up to a BVisomorphism thus we have

$$
i\left(a_{-2}\right)=u \cdot \alpha_{-2}, i\left(x_{-1}\right)=v \cdot \xi_{-1}, i\left(y_{2 n}\right)=w \cdot \zeta_{2 n}
$$

where $u, v, w \in \mathbb{Q}^{*}$. As $i$ is a morphism of BV-algebras we consider the equality $\Delta\left(i\left(x_{-1}\right)\right)=i\left(\Delta\left(x_{-1}\right)\right)$, from this equality we deduce that $v=1$. Finally we consider $i\left(\Delta\left(y_{2 n} x_{-1}\right)\right)=\Delta\left(i\left(y_{2 n} x_{-1}\right)\right)$,

$$
\begin{gathered}
i\left(\Delta\left(y_{2 n} x_{-1}\right)\right)=i\left(\mu^{\prime} y_{2 n}+\mu a_{-2}^{n} y_{2 n}^{2}\right)=w \mu^{\prime} \zeta_{2 n} \\
\Delta\left(i\left(y_{2 n} x_{-1}\right)\right)=\Delta\left(w \zeta_{2 n} \xi_{-1}\right)=w(-2 n-1) \zeta_{2 n} .
\end{gathered}
$$

thus $\mu^{\prime}=(-2 n-1)$.

In the next sections we end our computation by using the action of $S^{1}$ on the spaces of geodesics this gives us the value of $\lambda$ and $\mu$. 
2.2. A $S^{1}$-principal bundle for spaces of geodesics. - We consider the complex projective spaces equipped with the Fubini-Study metric. Let $\Sigma_{1}$ be the space of primitive closed geodesics $f: S^{1} \rightarrow \mathbb{C} P^{n}$. For an integer $q \geq 1$, we denote by $\Sigma_{q}$ the space of closed geodesics of the form $f(q t)$ for $f \in \Sigma_{1}$. As manifolds we have a canonical diffeomorphism $\Sigma_{1} \cong \Sigma_{q}$, one has a natural $S^{1}$-action on the spaces of $q$-iterated primitive geodesics $\Sigma_{q}$, of course this action depends of $q$. Then one has an action $\operatorname{Act}_{1}: S^{1} \times U \mathbb{C} P^{n} \rightarrow U \mathbb{C} P^{n}$ of $S^{1}$ on $U \mathbb{C} P^{n}$ given by the following diffeomorphism

$$
\begin{aligned}
D: \Sigma_{1} & \rightarrow U \mathbb{C} P^{n} \\
\gamma & \mapsto\left(\gamma(0), \gamma^{\prime}(0) /\left\|\gamma^{\prime}(0)\right\|\right) .
\end{aligned}
$$

We can also identify $\Sigma_{q}$ with $U \mathbb{C} P^{n}$ and we get another $S^{1}$-action denoted by Act $_{q}$, we get

$$
\operatorname{Act}_{q}(\theta, x)=\operatorname{Act}_{1}(q \theta, x)
$$

for any $\theta \in S^{1}$ an $x \in U \mathbb{C} P^{n}$. Since the isomorphism $\mathbb{E}_{k+1, *}^{1} \cong \mathbb{H}_{*}\left(B_{k+1}\left(\mathbb{C} P^{n}\right)\right)$ is compatible with the $\Delta^{\prime} \mathrm{s}, \Delta_{\mathrm{mss}}\left(\beta T^{2}\right)=2 \Delta(\beta T)$. On the other hand, $\Delta_{\mathrm{mss}}(\beta T)=\lambda a_{-2}^{n} T_{2 n}$ and $\Delta_{\mathrm{mss}}\left(\beta T^{2}\right)=\mu a_{-2}^{n} T_{2 n}^{2}$, we get the equality

$$
\mu=2 \lambda \text {. }
$$

Thus we focus on the case $q=1$ and on the action of $\Delta$ on $H_{*}\left(\Sigma_{1}, \mathbb{Z}\right) \cong$ $H_{*}\left(U \mathbb{C} P^{n}, \mathbb{Z}\right)$. The quotient of $U \mathbb{C} P^{n}$ by this action is denoted by $\Delta_{0}\left(\mathbb{C} P^{n}\right)$. This gives the $S^{1}$-equivariant fiber bundle $\rho: U \mathbb{C} P^{n} \rightarrow \Delta_{0}\left(\mathbb{C} P^{n}\right)$.

The authors of [16] compute the cohomology ring $H^{*}\left(\Delta_{0}\left(\mathbb{C} P^{n}\right)\right)$.

Lemma 2.3. - Corollary 3.3 of [16]. There is an isomorphism

$$
H^{*}\left(\Delta_{0}\left(\mathbb{C} P^{n}\right) ; \mathbb{Z}\right) \simeq \mathbb{Z}\left[x_{1}, x_{2}\right] /\left(Q_{n}, Q_{n+1}\right)
$$

with $\operatorname{deg}\left(x_{1}\right)=\operatorname{deg}\left(x_{2}\right)=2$ and $Q_{k}=\sum_{i=0}^{k} x_{1}^{k-i} x_{2}^{i}$.

By Poincaré duality, we deduce from this Lemma the intersection product ring algebra $\mathbb{H}_{*}\left(\Delta_{0}\left(\mathbb{C} P^{n}\right) ; \mathbb{Z}\right)$.

LEMMA 2.4. - There is an isomorphism

$$
\mathbb{H}_{*}\left(\Delta_{0}\left(\mathbb{C} P^{n}\right) ; \mathbb{Z}\right) \simeq \mathbb{Z}\left[y_{1}, y_{2}\right] /\left(Q_{n}, Q_{n+1}\right)
$$

with $\operatorname{deg}\left(y_{1}\right)=\operatorname{deg}\left(y_{2}\right)=-2$ and $Q_{k}=\sum_{i=0}^{k} y_{1}^{k-i} y_{2}^{i}$. 
2.3. The action of $\Delta$. - Let us recall the isomorphism of algebras:

$$
\mathbb{H}_{*}\left(U \mathbb{C} P^{n}\right) \cong \mathbb{Z}[\alpha, \beta] /\left(\alpha^{n+1}, \beta^{2},(n+1) \alpha^{n}, \alpha^{n} \beta\right)
$$

with $\operatorname{deg}(\alpha)=-2$ and $\operatorname{deg}(\beta)=-2 n-1$.

Proposition 2.5. $-\Delta(\beta)=\frac{(n+1)}{2} \alpha^{n}$ if $n$ is odd, otherwise it is equal to 0 , thus $\lambda=\frac{(n+1)}{2} \alpha^{n}$ if $n$ is odd, otherwise it is equal to 0

Proof of Proposition 2.5. - In order to compute $\Delta$ We use the fact that for any $S^{1}$-principal bundle

$$
S^{1} \rightarrow E \stackrel{p}{\rightarrow} B
$$

one has the formula

$$
\Delta=p_{!} \circ p_{*}: H_{*}(E) \rightarrow H_{*+1}(E) .
$$

First step. We compute $p_{*}(\beta)$. We use the shifted Serre spectral sequence $\mathbb{E}_{*, *}^{*}(\rho)$ of the fibration $\rho: U \mathbb{C} P^{n} \rightarrow \Delta_{0}\left(\mathbb{C} P^{n}\right)$. We use Poincaré duality to work multiplicatively with the cohomology structure. We have

$$
\mathbb{E}_{*, *}^{2}(\rho)=\mathbb{H}_{*}\left(S^{1}\right) \otimes \mathbb{H}_{*}\left(\Delta_{0}\left(\mathbb{C} P^{n}\right)\right) .
$$

Let us denote by $s \in \mathbb{H}_{-1}\left(S^{1}\right)$ a generator of $\mathbb{H}_{-1}\left(S^{1}\right)$. Since we know the $E^{\infty}$ term of this spectral sequence, we compute the differentials on the generators and deduce the other differentials by applying the Leibniz derivation rule. As we have

$$
\mathbb{H}_{*}\left(\Delta_{0}\left(\mathbb{C} P^{n}\right)\right) \cong \mathbb{Z}\left[y_{1}, y_{2}\right] /\left(Q_{n}, Q_{n+1}\right)
$$

the computation of differentials of the spectral sequence follows from the formula

$$
d_{2}(s \otimes 1)=1 \otimes\left(y_{1}-y_{2}\right) .
$$

This formula follows from the corollary 3.7 of [16]: we consider the Gysin exact sequence in cohomology, in low degree we have

$$
H^{0}\left(U \mathbb{C} P^{n}\right) \stackrel{\cup e u}{\rightarrow} H^{2}\left(\Delta_{0}\left(\mathbb{C} P^{n}\right)\right) \stackrel{p^{*}}{\rightarrow} H^{2}\left(U \mathbb{C} P^{n}\right) \stackrel{p^{!}}{\rightarrow} H^{1}\left(\Delta_{0}\left(\mathbb{C} P^{n}\right)\right)=0
$$

$1 \cup e u$ generates the kernel of $p^{*}$ which is equal by the corollary 3.7 of [16] to $\left(x_{1}-x_{2}\right)$ where $x_{i}$ are Poincaré dual to $y_{i}$, we conclude by identifying the cup product with the euler class with the $d_{2}$-differential. For degree reasons, the spectral sequence collapses at the $E^{3}$-page. There is no extension issues also for degree reasons.

Lemma 2.6. - The element $\sigma=s \otimes\left(\sum_{i=0}^{n}(i+1) y_{1}^{n-i} y_{2}^{i}\right) \in \mathbb{E}_{-2 n,-1}^{2}(\rho)$ represents $\beta$. 
Proof of Lemma 2.6. - Since $\sigma$ is a tensor product of $s$ with a unitary polynomial, it is a generator of the $\mathbb{Z}$-module $\mathbb{E}_{-2 n,-1}^{2}(\rho)$. To prove that it represents $\beta$, we only need to prove that it survives at the $E^{\infty}$ level that is to say $d_{2}(\sigma)=0$.

$$
\begin{aligned}
& d_{2}(\sigma)=d_{2}\left(s \otimes\left(\sum_{i=0}^{n}(i+1) y_{1}^{n-i} y_{2}^{i}\right)\right) \\
& =1 \otimes\left(y_{1}-y_{2}\right)\left(\sum_{i=0}^{n}(i+1) y_{1}^{n-i} y_{2}^{i}\right)
\end{aligned}
$$

and

$$
\begin{gathered}
\left(y_{1}-y_{2}\right)\left(\sum_{i=0}^{n}(i+1) y_{1}^{n-i} y_{2}^{i}\right)=\sum_{i=0}^{n+1} y_{1}^{n+1-i} y_{2}^{i}-(n+2) y_{2}^{n+1} \\
=Q_{n+1}-(n+2)\left(Q_{n+1}-y_{1} Q_{n}\right) .
\end{gathered}
$$

Then, $d_{2}(\sigma)$ lies in $\left(Q_{n}, Q_{n+1}\right)$. This ends the proof of Lemma 2.6.

From the preceding lemma we deduce that $p_{*}(\beta)=\sum_{i=0}^{n}(i+1) y_{1}^{n-i} y_{2}^{i}$.

Second step. In order to compute $p_{!}\left(p_{*}(\beta)\right)$ we use Poincaré duality, we have

$$
p_{!}=\mathbb{H}_{*}\left(\Delta_{0}\left(\mathbb{C} P^{n}\right)\right) \stackrel{D^{-1}}{\rightarrow} H^{*}\left(\Delta_{0}\left(\mathbb{C} P^{n}\right)\right) \stackrel{p^{*}}{\rightarrow} H^{*}\left(U \mathbb{C} P^{n}\right) \stackrel{D}{\rightarrow} \mathbb{H}_{*}\left(U \mathbb{C} P^{n}\right)
$$

from the Gysin exact sequence we know that $p^{*}\left(x_{1}\right)=p^{*}\left(x_{2}\right)$ and that $p^{!}\left(D^{-1}(\alpha)\right) \in H^{1}\left(\Delta_{0}\left(\mathbb{C} P^{n}\right)\right)=0$. Thus we get that $p^{*}\left(x_{1}\right)=p^{*}\left(x_{2}\right)=D^{-1}(\alpha)$ and that

$$
p_{!}\left(p_{*}(\beta)\right)=\sum_{i=0}^{n}(i+1) p_{!}\left(y_{1}^{n-i}\right) p_{!}\left(y_{2}^{i}\right)=\left(\sum_{i=0}^{n}(i+1)\right) \alpha^{n}
$$

\section{BIBLIOGRAPHY}

[1] R. Botт - "Lectures on Morse theory, old and new", Bull. Amer. Math. Soc. (N.S.) 7 (1982), p. 331-358.

[2] M. CADEK \& Z. MoRAVEK - "Loop homology of quaternionic projective spaces", preprint arXiv:math.AT/10041550.

[3] M. ChAS \& D. Sullivan - "String topology", preprint arXiv:math.GT/9911159.

[4] R. L. Cohen \& J. D. S. Jones - "A homotopy theoretic realization of string topology", Math. Ann. 324 (2002), p. 773-798.

[5] R. L. Cohen, J. D. S. Jones \& J. YAN - "The loop homology algebra of spheres and projective spaces", in Categorical decomposition techniques in algebraic topology (Isle of Skye, 2001), Progr. Math., vol. 215, Birkhäuser, 2004, p. 77-92. 
[6] Y. FÉlix \& J.-C. Thomas - "Rational BV-algebra in string topology", Bull. Soc. Math. France 136 (2008), p. 311-327.

[7] Y. Felix, J.-C. Thomas \& M. Vigué-Poirrier - "The Hochschild cohomology of a closed manifold", Publ. Math. Inst. Hautes Études Sci. 99 (2004), p. 235-252.

[8] M. Gerstenhaber - "The cohomology structure of an associative ring", Ann. of Math. 78 (1963), p. 267-288.

[9] E. Getzler - "Batalin-Vilkovisky algebras and two-dimensional topological field theories", Comm. Math. Phys. 159 (1994), p. 265-285.

[10] M. Goresky \& N. Hingston - "Loop products and closed geodesics", Duke Math. J. 150 (2009), p. 117-209.

[11] R. HEPWORTH - "String topology for complex projective spaces", preprint arXiv:math.AT/0908.1013.

[12] F. LAudenbach - "A note on the Chas-Sullivan loop product", preprint arXiv:math.GT/0903.2801.

[13] L. MENICHI - "Batalin-Vilkovisky algebras and cyclic cohomology of Hopf algebras", K-Theory 32 (2004), p. 231-251.

[14] , "Batalin-Vilkovisky algebra structures on Hochschild cohomology", Bull. Soc. Math. France 137 (2009), p. 277-295.

[15] _ "String topology for spheres", Comment. Math. Helv. 84 (2009), p. $135-157$.

[16] I. Ottosen \& M. BöKStedT - "String cohomology groups of complex projective spaces", Algebr. Geom. Topol. 7 (2007), p. 2165-2238.

[17] H. TAmanoI - "Batalin-Vilkovisky Lie algebra structure on the loop homology of complex Stiefel manifolds", Int. Math. Res. Not. 2006 (2006), Art. ID 97193, 23.

[18] T. TRAdLER - "The Batalin-Vilkovisky algebra on Hochschild cohomology induced by infinity inner products", Ann. Inst. Fourier (Grenoble) 58 (2008), p. 2351-2379.

[19] T. YANG - "A Batalin-Vilkovisky algebra structure on the Hochschild cohomology of truncated polynomials", preprint arXiv:0707.4213.

[20] W. ZILLER - "The free loop space of globally symmetric spaces", Invent. Math. 41 (1977), p. 1-22. 